\title{
Interactive comment on "Hydrological Modeling in an Ungauged Basin of Central Vietnam Using SWAT Model” by A. Rafiei Emam et al.
}

\section{A. Rafiei Emam et al.}

rafiei99@gmail.com

Received and published: 3 May 2016

The authors would like to appreciate the reviewer's constructive criticism on the manuscript. In the following pages the authors have replied to each comment and explained how the manuscript will be modified based on these constructive comments:

\#General comment: This paper presents a standard application of the SWAT model for a watershed in central Vietnam. It is not clear and also not communicated by the authors, what the novel aspects of this study are. Any revised version should clearly communicate the novelty of the application and show how it goes beyond the state of the art.

@General answer: As a response to the reviewer's comment, the novelty of this paper is the calibration procedures in which were done in three steps instead of using just 
one step (e.g. river discharge calibration as usual). The other novelty is to use MODIS data in order to calibrate the ETa in the study area. We believe that in the absence of the river discharge data, ETa can parameterize the model in high precision.

\# comment 1. Essentially, this paper compares two models to estimate river discharge for an ungauged catchment (catchment 1): Model 1, called "regionalization approach" here, multiplies observed river discharge from a neighboring catchment (catchment 2) with the ratio of the two catchment areas (catchment 1 area/catchment 2 area). Model 2 is the SWAT model. Subsequently, Model 1 is taken as the truth and Model 2 is calibrated to reproduce that postulated truth. This approach is questionable. Normally, one would expect model 2 to perform better than model 1 (otherwise - why build it?), so why calibrate it to match model 1 ? A better approach may have been to set up and calibrate the SWAT model for both catchment 1 and catchment 2 and then transfer calibrated parameters from catchment 2 to catchment 1 .

@Answer 1: Our target area is Aluoi in central Vietnam which doesn't have measured data (river discharge). We interested in evaluating the water resources behavior in this area using hydrological model. However, to calibrate our model we predicted the river data in this area by ratio method using nearby measured data. We are not comparing two models in two basins, or in the other hands the two models are not comparable. We predicted the river discharge at the outlet of the Aluoi basin by using measured data available in nearby stations. Because the characteristics of two basins are the same (Table 1) therefore converting river discharge from one basin to another basin by ratio method is acceptable. However, as you suggested for transferring parameters (from model 1 to model 2), instead of transferring river discharge data, we have done this additional part and the manuscript was revised based on that. Therefore the ratio method was eliminated from the procedure of calibration.

Printer-friendly version

\# comment 2. The calibration strategy is unclear to me. Three types of data area used: River discharge, actual ET and crop yield. Calibration against these targets is done sequentially, but I expect that a number of SWAT parameters will be sensitive to more 
than one of these targets. How are you dealing with trade-offs between the targets? A formal multi-objective calibration approach would probably have been appropriate.

@Answer 2: We agree with the reviewer in his/her point. As the reviewer mentioned some of the parameters might be sensitive to more than one objective. In order to overcome this issue, we used the best range of values instead of an absolute value for each parameter. For instance we calibrated the parameters for river discharge, then we fixed the parameters in a range (instead of single value), and added the relevant parameters of the second objective (i.e. ETa). Same as the first step, we defined again the best range for each parameter and then added the third set of parameters (i.e. for crop calibration). With this strategy we overcame the trade-off between the targets. Additionally, we calibrated the parameters mostly in the HRU level, it means that for each target even the same parameters could parameterize separately based on soil, land use, management practice and HRU level.

\# comment 3. P1, Line 29 and following: This states the main motivation for the study. However, to me this reads like a rationale for a consulting project, not for a scientific research paper.

$@$ @nswer 3: The central Vietnam and especially Aluoi district is under severe pressure of changing hydrological behavior due to climate change and human induced factors. Therefore, we just try to explain why we are going to study this area. Therefore we disagree with the reviewer for this comment.

\# comment 4. Calibration with actual ET from MODIS: How was this done? At the HRU level or catchment level? How did you make the match between grid-based MOD-16 and SWAT? I guess in eq 2 ETa would be better symbology than $Q$.

@Answer 4: The calibration of ETa was done in sub-basin level. More than $90 \%$ of the area covered by forests. We overlapped MOD16 images with land use and subbasin maps, then we selected some the sub-basins with fully covered by forests within each of MOD16 cells, and finally the ETa in these area was extracted. We should

Printer-friendly version

Discussion paper 
mention that the watershed was delineated into 20 sub-basins. We agree with the reviewer for changing the $Q$ symbology to ETa. As in this step we are talking about evapotranspiration. The symbol was revised in the updated version.

Details \# comment 2-1. P1, Line 18: Nash-Sutcliffe

@Answer 2-1: Was edited.

Interactive

comment

\# comment 2-2. P1,Line 24: hydraulic models

@Answer 2-2: Was edited.

\# comment 2-3. P3, Line 23: "MODIS time series data" is too unspecific. Please state which MODIS products have been used. Are these potential ET or actual ET products or both?

@Answer 2-3: This sentence was revised. We used both potential and actual evapotranspiration. ftp://ftp.ntsg.umt.edu/pub/MODIS/NTSG_Products/MOD16/

\# comment 2-4. P3, Line 31: "slope"

@Answer 2-4: Was edited.

\# comment 2-5. Eq 1: I guess Qgw is capillary rise of water back into the soil zone, so should be +Qgw, not -Qgw. The term "soil infiltration" for wseep may not be optimal. "Deep percolation" may be better. ETa: Please always specify if this is reference, potential or actual ET. I guess in this case it is actual ET.

$@$ Answer 2-5: The definitions of parameters were modified. The water balance equation (Eq1) is used from the SWAT documentation. We just tried to rewrite the definition, but you are completely right. Wseep is the amount of percolation, ET is actual evapotranspiration. The Qgw as mentioned in the SWAT documentation is return flow. Here returns flow is base flow. I refer you to the page 98 of the SWAT theoretical documentation (http://swat.tamu.edu/media/99192/swat2009-theory.pdf ). 
\# comment 2-6. Please use continuous line numbering throughout the paper to facilitate the review Process

@Answer 2-6: We changed the line numbers through the paper based on the continuous lines numbers.

\# comment 2-7. P5, Line 10ff: Here the term "regionalization" is used for parameter transfer between catchments, while further up it is used for what is now called "ratio method". Please be consistent.

@Answer 2-7: We just tried to explain what is behind the regionalization, for those readers who are not familiar with this term. However, as the reviewer suggested we changed our approach of regionalization, as I explained earlier (comment 1).

\# comment 2-8. P6, Line 1: "thickness" should be "width", you could also use the term "sharpness".

@Answer 2-8: was edited.

\# comment 2-9. Page 8, Line 25f: This procedure for dealing with the bias in the MODIS potential ET sounds very "ad hoc". Should be justified better.

@Answer 2-9: As MODIS products are globally scaled and they have coarse resolution, data should be evaluated in case of accuracy before using in the research studies. As we have enough data to estimate ETP, we compared our results of ETP with those obtained by MODIS product (i.e. MOD16). We understood that the dynamic of MOD16 is correct but the absolute values are not match with our data. Therefore, we multiplied a constant value, obtained by trial and error, in MOD16 data to overcome the bias issues.

Interactive comment on Hydrol. Earth Syst. Sci. Discuss., doi:10.5194/hess-2016-44, 2016. 